\title{
Minireview \\ CDK-independent activities of D type cyclins
}

\author{
René Bernards * \\ Division of Molecular Carcinogenesis and Center for Biomedical Genetics, Netherlands Cancer Institute, Plesmanlaan 121, \\ 1066 CX Amsterdam, The Netherlands
}

Received 29 June 1999; accepted 31 August 1999

Keywords: Cyclin D1; Cell cycle; Estrogen receptor; Myb proteins; Breast cancer

\section{Contents}

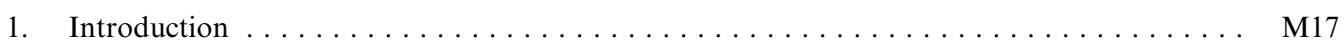

2. The cyclin D1-estrogen receptor connection in breast cancer ............ M18

3. Interactions with Myb-like proteins $\ldots \ldots \ldots \ldots \ldots \ldots \ldots \ldots \ldots \ldots \ldots$

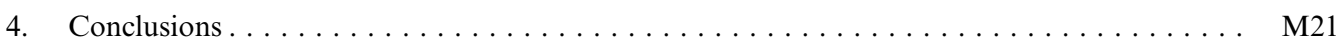

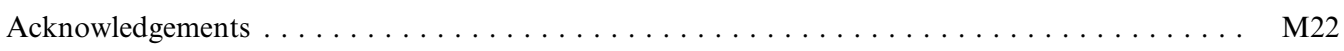



\section{Introduction}

D type cyclins are major downstream targets of extracellular signaling pathways which act to transduce mitogenic signals to the cell cycle machinery. Transcriptional induction of $\mathrm{D}$ type cyclins occurs in response to a wide variety of mitogenic stimuli, including the Ras signaling cascade $[1,2]$ and the APC- $\beta$-catenin-Tcf/Lef pathway [3]. In addition, cyclin D1 protein turnover and subcellular localization are highly regulated during the cell cycle [4]. Because of their critical role in linking cytoplasmic signals to nuclear responses it is perhaps not surprising that $\mathrm{D}$ type cyclins are frequent targets of mutagenesis in various types of cancer [5].

Biochemically, D-type cyclins act, in part, as regulatory subunits of cyclin-dependent kinases (CDKs)

\footnotetext{
* Fax: +31-20-512-1954; E-mail: bernards@nki.nl
}

4 and 6. Cyclin D/CDK4/6 complexes, together with cyclin E/CDK2, cause phosphorylation of the retinoblastoma family of tumor suppressor proteins $(\mathrm{pRb}$, p107 and p130) in the G1 phase of the cell cycle, resulting in abrogation of their growth-inhibitory activity (see [6] for a review). The ability of D type cyclins to interact with and activate their CDK partners is antagonized by the p16 $6^{\mathrm{INK} 4}$ family of CDK inhibitors. Mutations in $p 16^{I N K 4 A}$ are found in a variety of spontaneous tumors and heterozygosity for p16 $6^{I N K 4 A}$ in the germ line predisposes to melanoma [7]. It is generally believed that this $\mathrm{p} 16^{\mathrm{INK} 4 \mathrm{~A}}$-cyclin $\mathrm{D} 1-\mathrm{pRb}$ pathway is deregulated in virtually all human cancers and this notion underscores the critical role of D type cyclins as CDK regulators in growth control.

However, in the past 2 years it has become apparent that $\mathrm{D}$ type cyclins also have activities that are unrelated to their function as CDK regulatory subunits. These novel CDK-independent activities of D type 


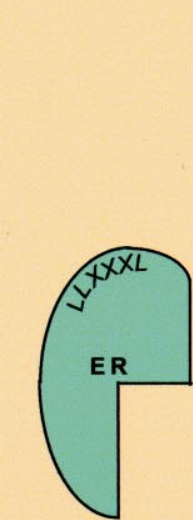

Inactive

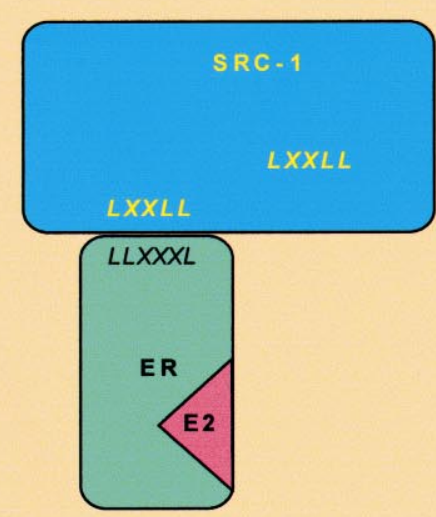

Partially active

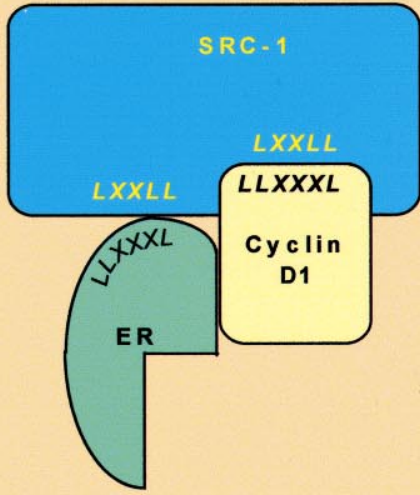

Partially active



Fully active

A

B

D

Fig. 1. Multiple ways to activate the ER. In the absence of ligand, ER is unable to interact with SRCs directly as its leucine-rich coactivator interaction motif (AF2, indicated as LLXXXL) is sterically unavailable for SRC interaction. (A) Hormone binding by ER exposes AF2 and allows recruitment of SRCs to ER, leading to activation of ER. (B) Hormone-independent binding of cyclin D1 to ER provides a single leucine-rich interaction motif for SRCs on the cyclin D1/ER complex, which is present in the carboxyl terminus of cyclin D1. This results in partial activation of ER. (C) Ligand binding of ER in the presence of cyclin D1 provides two independent interaction surfaces for SRCs: one is formed by the leucine-rich motif in AF-2 of ER and a second in the carboxyl terminus of cyclin D1. (D) The observed synergism between estrogen and cyclin D1 in ER activation may result from their cooperative recruitment of SRCs to the ER. The protein interaction motifs are shown in italics ( $\mathrm{L}=$ leucine, $\mathrm{X}=$ any amino acid).

cyclins and their possible contribution to growth regulation and cancer are the topic of this brief review.

\section{The cyclin D1-estrogen receptor connection in breast cancer}

Cyclin D1 is either amplified or over-expressed in a number of human malignancies, the most prominent being breast cancer, in which up to $50 \%$ of all cases have elevated levels of cyclin D1 [8-10]. The relevance of cyclin DI over-expression in breast cancer is further emphasized by the finding that tissue-specific transgenic expression of cyclin D1 in mice results in mammary hyperplasia and adenocarcinoma [11]. Consistent with a prominent role for cyclin D1 in growth of breast epithelium, cyclin D1 knockout mice show a marked defect in breast epithelium development during pregnancy $[12,13]$.

Estrogens are major determinants of proliferation of breast epithelial cells. The mitogenic activity of estrogens is mediated through the estrogen receptor (ER), a nuclear hormone receptor that acts as a ligand-regulated transcription factor. Interestingly, whereas only some $50-60 \%$ of human breast cancers express a functional ER, cyclin D1 over-expression is seen preferentially in ER-positive breast cancers $[10,14]$. There are several possible explanations for the observed co-expression of cyclin D1 and ER in breast cancer. Since activation of the ER by hormone leads to transcriptional induction of cyclin D1 [15], it is possible that cyclin D1 expression merely reflects the presence of a functional ER that drives cyclin D1 expression in the tumor cells. Alternatively, it is possible that cyclin D1 derives (part of) its oncogenic activity in breast cancer by acting through the ER. In this scenario, cyclin D1 expression would confer a selective advantage in breast cancer only when a functional ER is present. This latter notion is supported by two recent studies which indicate that expression of cyclin D1 leads to hormone-independent activation of ER [16,17]. Surprisingly, this effect of cyclin D1 on ER does not require $\mathrm{CDK}$ binding by cyclin $\mathrm{D} 1$, since it can be recapitulated with the cyclin D1 K112E mutant, which is unable to bind CDK4. In fact, a cyclin D1/CDK4 complex is unable to activate the ER, providing further support for the notion that cyclin 
D1 acts on ER in a CDK-independent fashion. Subsequent experiments showed that cyclin D1 forms a direct physical complex with the ER [16,17]. Importantly, cyclin D1 not only interacts with the ER but also with several members of a family of related steroid receptor coactivators (SRCs) $[18,19]$.

When ER is activated by ligand, a dramatic conformational change takes place which exposes a short helical motif (named AF2) that constitutes a binding site for the SRC family of coactivators (Fig. 1A,B). It is generally believed that SRC recruitment to ER is responsible for transcriptional activation of ER. Interestingly, the motif of cyclin D1 that mediates binding to SRCs is very similar to the AF2 motif used by ER to bind SRCs [18]. Thus, the finding that cyclin D1 can bind both ER and SRCs suggests that cyclin D1 can act as a bridging factor that recruits coactivators to ER in the absence of hormone (Fig. 1C). If cyclin D1 and hormone are both present, two different contacts are made between ER and its coactivator (Fig. 1D). Consistent with this model, a strong synergy is seen between cyclin D1 and estrogen in activation of ER [16].

The mechanism of ER activation by cyclin D1 may well have clinical ramifications. As the binding of cyclin D1 to both ER and SRCs does not require estrogen, it is possible that a cyclin D1-activated ER is insensitive to the actions of anti-estrogens such as tamoxifen (which inhibits ER activity by competitive binding to the hormone binding site on ER). Antiestrogens are widely used in the clinic for the treatment of ER-positive breast cancers, but some $25 \%$ of ER positive tumors fail to respond to anti-estrogens for as yet unknown reasons. Indeed, in short term assays, ectopic expression of cyclin D1 appears to override the effects of tamoxifen on ER activity and cell cycle progression [16,20]. However, in long term growth inhibition assays, cyclin D1 does not appear to override the cytostatic effects of tamoxifen [21]. It is, therefore, not clear at present whether cyclin D1 expression contributes to resistance to anti-estrogens in ER-positive breast cancers.

The finding that cyclin D1 can activate ER through direct binding provides clear evidence that cyclin D1 can act as an upstream regulator of ER activity. However, two lines of experimental evidence place cyclin DI downstream of ER. First, activation of ER leads to increased cyclin D1 transcription and indeed an estrogen-responsive element has been found in the cyclin D1 promoter [15,22]. Consistent with this, anti-estrogens reduce cyclin D1 expression in breast cancer cells [23]. Second, hormone activation of ER leads to activation of the Src/Ras/MAPK pathway [24,25]. Since cyclin Dl is downstream of MAPK [26], this pathway constitutes a second, independent, mechanism of induction of cyclin D1 expression by ER. Together, these data suggest the existence in breast cancers of an autostimulatory loop in which activation of ER by hormone leads to transcriptional induction of cyclin D1. This newly synthesized cyclin D1 protein can then bind to ER to cause further ER activation. In this way cyclin D1 and estrogen may act synergistically in stimulation of breast cancer growth. Such an autostimulatory loop that involves both ER and cyclin D1 may well explain why co-expression of ER and cyclin D1 is seen in many breast cancers.

\section{Interactions with Myb-like proteins}

The first indication that cyclin D can modulate the function of $\mathrm{Myb}$ transcription factors stems from 1996 when a protein with Myb-like repeats, named DMP1, was isolated in a two hybrid screen with cyclin D2 as a bait [27]. DMP1 has all the hallmarks of a transcription factor, since it can bind to DNA in a sequence-specific fashion and activate transcription. Cyclin D2 interacts with the DNA binding domain of DMP1, thereby interfering with DMP1 DNA binding. Importantly, the cyclin D1 K112E mutant that cannot bind CDK4 can still interact with DMP1 and inhibit its activity, indicating that the effect of D type cyclins on DMP1 is, again, CDK-independent. Consistent with this, cyclin D2 forms mutually exclusive complexes with CDK4 and DMP1, since no ternary cyclin/CDK/DMP1 complexes can be detected [27]. Interest in DMP1 and its interaction with cyclin D increased when it was found that, in transfected cells, DMP1 expression prevents S phase entry. DMP1-induced growth arrest can be overridden by the cyclin D1 K112E mutant, indicating that the cyclin D1-induced override of the DMP1-induced growth arrest is also CDK-independent [28]. Recent data indicate that DMP1 binding sites are found in the promoters of 


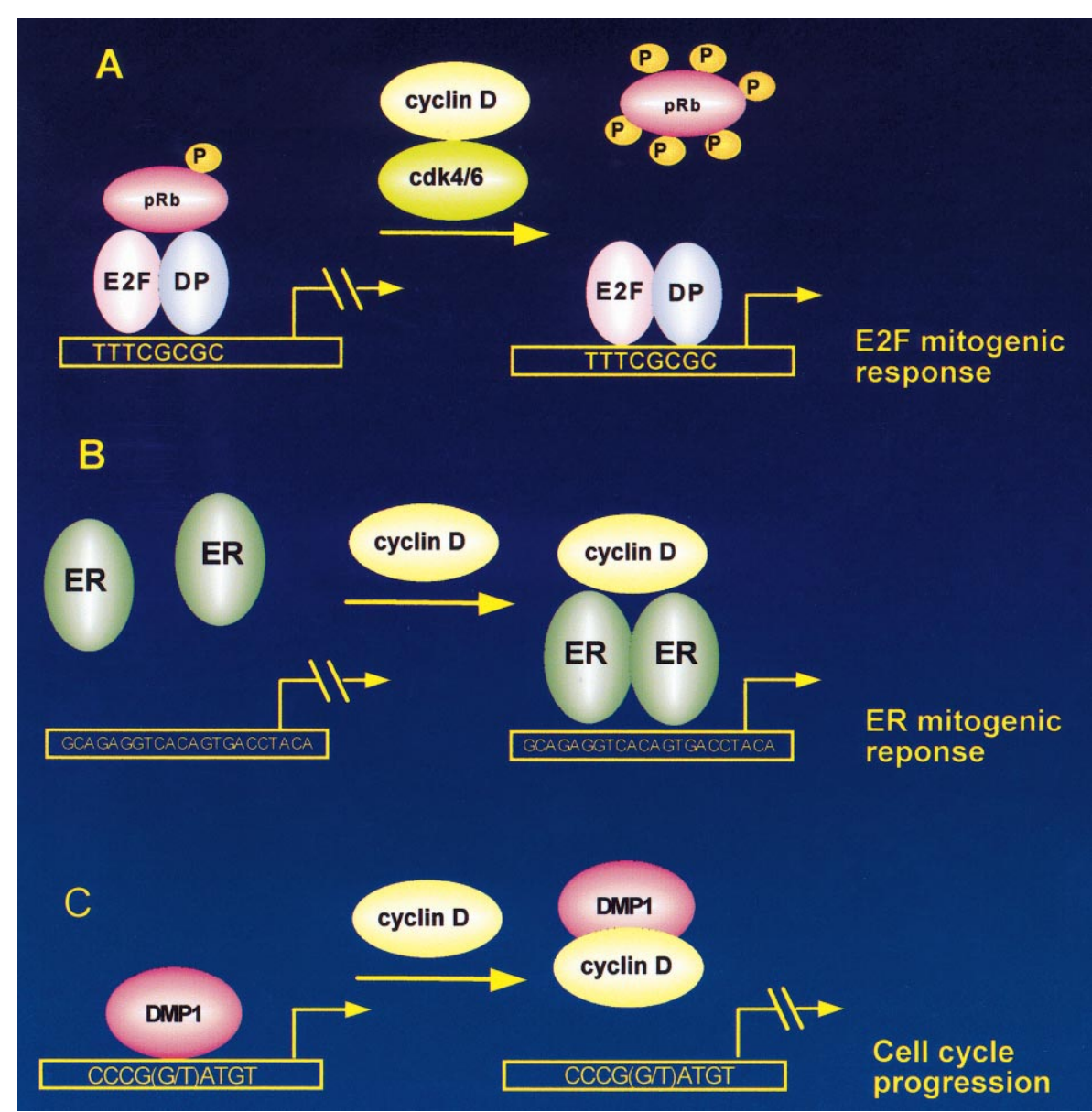

Fig. 2. Three mechanisms of action of D type cyclins. (A) CDK-dependent phosphorylation of pRb by cyclin D leads to release of active E2F transcription factors that drive cells into S phase. (B) CDK-independent binding of cyclin D1 to the ER induces activation of ER in the absence of hormone, leading to a mitogenic response. (C) CDK-independent binding of cyclin D to DMP1 inhibits the growth-restraining activity of DMP1, thereby allowing progression through the cell cycle.

at least two cellular genes, CD13/Aminopeptidase N and the p19 arf tumor suppressor gene $[29,30]$. Importantly, DMP1 does not induce cell cycle arrest in p19arf null cells, indicating that $p 19^{a r f}$ is a critical downstream target of DMP1 [30]. The finding that the human DMP1 gene maps to chromosome $7 \mathrm{q} 21$, a region frequently deleted in myeloid leukemia, suggests that DMP1 has an important role in normal growth regulation and may contribute to tumorigenesis when mutated [31].

Apart from DMP1, the viral Myb protein, but not its cellular homologue, is subject to negative regulation by D type cyclins [32]. As was seen for DMP1, the effects of D type cyclins on $\mathrm{v}-\mathrm{Myb}$ are CDKindependent and result from an interaction of the cyclin with the v-Myb DNA binding domain. At first glance it appears counter-intuitive that an oncogenic transcription factor like v-Myb would be subject to negative regulation by a growth-promoting cyclin. However, in addition to their transactivating capacity, both c-Myb and v-Myb can also function as repressors of transcription [33]. In this respect, $\mathrm{Myb}$ proteins may resemble $\mathrm{E} 2 \mathrm{~F}$ transcription factors, which can also both activate and repress transcription. As was discussed in this journal recently, the exact balance between repression and transactivation by E2F appears critical for the effects that E2Fs have on cellular physiology [34]. If this analogy also holds true for Myb proteins, D type cyclins could act to shift the balance from $\mathrm{v}-\mathrm{Myb}$ transactivation to repression. In this respect, it is noteworthy that binding of cyclin D to v-Myb does not affect v-Myb DNA binding which may allow the $\mathrm{v}-\mathrm{Myb} /$ cyclin $\mathrm{D}$ complex to maintain its repressor function, whereas bind- 


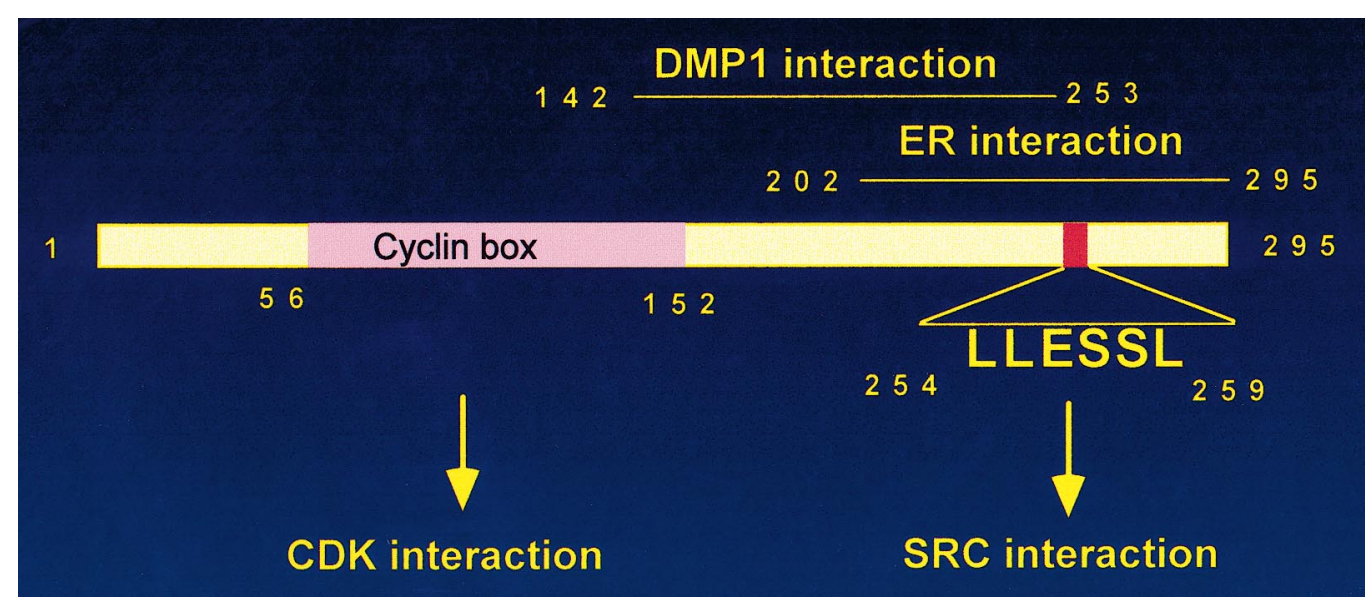

Fig. 3. Protein interaction surfaces on cyclin D1. The amino terminal half of cyclin D1 harbors the highly conserved cyclin box, which constitutes the interaction site for the CDKs. The carboxyl-terminal half contains binding sites for the ER (amino acid 202-295), SRCs (amino acids 254-259) and the Myb-like transcription factor DMP1 (amino acids 142-253).

ing of cyclin D to DMP1 does interfere with DMP1 DNA binding $[28,32]$.

\section{Conclusions}

D type cyclins can have CDK-independent interactions with two, distinct types of transcription factors. They can enhance the growth-promoting activity of the ER through recruitment of coactivators and inhibit the growth-restraining capacity of the Myb-like transcription factor, DMP1 (Fig. 2). Both effects of D type cyclins are mediated through direct binding, and these protein interaction surfaces reside in the carboxyl terminal half of cyclin D1, a region that is distinct from the well-defined CDK interaction surface (Fig. 3). In spite of this, cyclin D1 cannot activate ER when in complex with a CDK partner [16]. Similarly, no cyclin D/CDK/DMP1 trimeric complexes can be detected and effects of cyclin D1 on $\mathrm{v}-\mathrm{Myb}$ are counteracted by CDK4 expression [32]. All these data suggest that cyclin D enters into mutually exclusive complexes with either a CDK or a transcription factor like Myb or ER. The finding that CDK co-expression readily abrogates the effects of cyclin D1 on ER suggests that cyclin D1/ER complexes can only form when a CDK partner is unavailable and the same may very well hold true for the cyclin D/Myb interaction. This begs the question of whether such interactions can ever take place under physiological conditions, since, in most cell types, the CDK is more highly expressed than the cyclin component. Even though little is known about this at present, it appears that in the differentiating breast epithelium, it is possible for cyclin D1 to exist in molar excess over its CDK partner. When murine SCp2 breast epithelial cells are induced to differentiate into cells having features of alveolar milk-secreting cells, expression of cyclin D1 is upregulated, while CDK4 is down-modulated. Concomitantly, the activity of ER is enhanced [17]. This suggests that, in the differentiating breast epithelium, cyclin D1 is made available to ER to enhance its activity. In a pathologic situation where cyclin D1 is highly over-expressed, as is seen in many breast cancers, such cyclin D/ER complexes may form more readily, even in the presence of moderate levels of CDK4/6.

An important question that remains to be answered is how relevant the CDK-independent activities of D type cyclins are in normal growth control and in cancer. The observation that cyclin D1 knockout mice have clear defects in breast epithelium proliferative responses during pregnancy indicates that cyclin D1 has a clear role in this process. However, these experiments do not address whether cyclin D1 is required in the breast as a CDK regulator or as an ER activator. The finding that the breast epithelium defect of cyclin D1 knockout mice can be rescued by expression of cyclin $\mathrm{E}$ (which does not activate ER) may indicate that cyclin D1 does not have a major role in ER regulation in the developing breast [35]. 
Alternatively, cyclin E may rescue the growth defect that results from loss of cyclin D1 in the breast through an independent, yet unknown pathway that compensates for loss of ER activation by cyclin D1. Finally, it should be kept in mind that these CDK-independent activities of D type cyclins were identified in transfection experiments in which proteins are highly expressed. It is, therefore, possible that the observed, CDK-independent effects of cyclin $\mathrm{D}$ do not play a major role in normal physiology, but rather in pathological (cancerous) situations in which the cyclins are expressed at a high level. The availability of specific mutants of cyclin D1 that are wild-type for CDK activation but mutant for ER activation [18] may help resolve the exact contribution of cyclin D1 to ER activation in normal breast development and breast cancer.

\section{Acknowledgements}

I thank Daniel Peeper for critical reading of this manuscript, Charles Sherr and Piotr Sicinski for communicating data prior to publication. The work of the author is supported by grants from the Dutch Cancer Society.

\section{References}

[1] D.S. Peeper, T.M. Upton, M.H. Ladha, E. Neuman, J. Zalvide, R. Bernards, J.A. DeCaprio, M.E. Ewen, Nature 386 (1997) 177-181.

[2] D.S. Peeper, R. Bernards, Febs Lett. 410 (1997) 11-16.

[3] O. Tetsu, F. McCormick, Nature 398 (1999) 422-426.

[4] J.A. Diehl, M. Cheng, M.F. Roussel, C.J. Sherr, Genes Dev. 12 (1998) 3499-3511.

[5] C.J. Sherr, Science 274 (1996) 1672-1677.

[6] R. Bernards, Biochim. Biophys. Acta Rev. Cancer 1333 (1997) M33-M40.

[7] A. Kamb, N.A. Gruis, F.J. Weaver, Q. Liu, K. Harshman, S.V. Tavtigian, E. Stockert, R.r. Day, B.E. Johnson, M.H. Skolnick, Science 264 (1994) 436-440.

[8] M.F. Buckley, K.J. Sweeney, J.A. Hamilton, R.L. Sini, D.L. Manning, R.I. Nicholson, A. deFazio, C.K. Watts, E.A. Musgrove, R.L. Sutherland, Oncogene 8 (1993) 2127-2133.

[9] E. Schuuring, E. Verhoeven, H. van Tinteren, J.L. Peterse, B. Nunnink, F.B. Thunnissen, P. Devilee, C.J. Cornelisse, M. van de Vijver, W.J. Mooi et al., Cancer Res. 52 (1992) 5229-5234.

[10] P.J. van Diest, R.J. Michalides, L. Jannink, P. van der Valk, H.L. Peterse, J. de Jong, C.J. Meijer, J.P. Baak, Am. J. Pathol. 150 (1997) 705-711.
[11] T.C. Wang, R.D. Cardiff, L. Zukerberg, E. Lees, A. Arnold, E.V. Schmidt, Nature 369 (1994) 669-671.

[12] P. Sicinski, J.L. Donaher, S.B. Parker, T. Li, A. Fazeli, H. Gardner, S.Z. Haslam, R.T. Bronson, S.J. Elledge, R.A. Weinberg, Cell 82 (1995) 621-630.

[13] V. Fantl, G. Stamp, A. Andrews, I. Rosewell, C. Dickson, Genes Dev. 9 (1995) 2364-2372.

[14] C. Gillett, P. Smith, W. Gregory, M. Richards, R. Millis, G. Peters, Int. J. Cancer 69 (1996) 92-99.

[15] M. Planas-Silva, R.A. Weinberg, Mol. Cell. Biol. 17 (1997) 4059-4069.

[16] R.M.L. Zwijsen, E. Wientjens, R. Klompmaker, J. van der Sman, R. Bernards, R.J.A.M. Michalides, Cell 88 (1997) 405-415.

[17] E. Neuman, M.H. Ladha, N. Lin, T.M. Upton, S.J. Miller, J. DiRenzo, R.G. Pestell, P.W. Hinds, S.F. Dowdy, M. Brown, M.E. Ewen, Mol. Cell. Biol. 17 (1997) 5338-5347.

[18] R.M. Zwijsen, R.S. Buckle, E.M. Hijmans, C.J. Loomans, R. Bernards, Genes Dev. 12 (1998) 3488-3498.

[19] C. McMahon, T. Suthiphongchai, J. DiRenzo, M.E. Ewen, Proc. Natl. Acad. Sci. USA 96 (1999) 5382-5387.

[20] N.R. Wilcken, O.W. Prall, E.A. Musgrove, R.L. Sutherland, Clin. Cancer Res. 3 (1997) 849-854.

[21] C. Pacilio, D. Germano, R. Addeo, L. Altucci, V.B. Petrizzi, M. Cancemi, L. Cicatiello, S. Salzano, F. Lallemand, R.J. Michalides, F. Bresciani, A. Weisz, Cancer Res. 58 (1998) 871-876.

[22] L. Altucci, R. Addeo, L. Cicatiello, S. Dauvois, M.G. Parker, M. Truss, M. Beato, V. Sica, F. Bresciani, A. Weisz, Oncogene 12 (1996) 2315-2324.

[23] C.K. Watts, K.J. Sweeney, A. Warlters, E.A. Musgrove, R.L. Sutherland, Breast Cancer Res. Treat. 31 (1994) 95105.

[24] A. Migliaccio, D.M. Di, G. Castoria, F.A. de, P. Bontempo, E. Nola, F. Auricchio, EMBO J. 15 (1996) 1292-1300.

[25] G. Castoria, M.V. Barone, D.M. Di, A. Bilancio, D. Ametrano, A. Migliaccio, F. Auricchio, EMBO J. 18 (1999) $2500-2510$.

[26] J.N. Lavoie, G. L'Allemain, A. Brunet, R. Muller, J. Pouyssegur, J. Biol. Chem. 271 (1996) 20608-20616.

[27] H. Hirai, C.J. Sherr, Mol. Cell. Biol. 16 (1996) 6457-6467.

[28] K. Inoue, C.J. Sherr, Mol. Cell. Biol. 18 (1998) 1590-1600.

[29] K. Inoue, C.J. Sherr, L.H. Shapiro, J. Biol. Chem. 273 (1998) 29188-29194.

[30] K. Inoue, M.F. Roussel, C.J. Sherr, Proc. Natl. Acad. Sci. USA 96 (1999) 3993-3998.

[31] S.M. Bodner, C.W. Naeve, K.M. Rakestraw, B.G. Jones, V.A. Valentine, M.B. Valentine, F.W. Luthardt, C.L. Willman, S.C. Raimondi, J.R. Downing, M.F. Roussel, C.J. Sherr, A.T. Look, Gene 229 (1999) 223-228.

[32] B. Ganter, S.1. Fu, J.S. Lipsick, EMBO J. 17 (1998) 255-268.

[33] B. Ganter, J.S. Lipsick, Oncogene 15 (1997) 193-202.

[34] L. Yamasaki, Biochim. Biophys. Acta 1423 (1999) M9-15.

[35] Y. Geng, W. Whoriskey, M.Y. Park, R.T. Bronson, R.H. Medema, L. Tiansen, R.A. Weinberg, P. Sicinski, Cell 97 (1999) 767-777. 